\title{
Morphological and molecular characterization of Curvularia and related species associated with leaf spot disease of rice in Peninsular Malaysia
}

\begin{abstract}
Curvularia species are important phytopathogens reported worldwide. They are closely related; consist of major destructive pathogens mainly for grasses and cereal plants including rice (Oryza sativa). A leaf spot symptom of rice is one of the common symptoms found in the rice field and caused reduction of rice yield. However, there are no reports on Curvularia species associated with rice leaves showing spot symptoms. The objectives are to isolate and characterize Curvularia and related species from leaf spot of rice by using morphological and molecular characterization and to determine the phylogenetic relationship between the isolated fungi. Fungal isolation was done from diseased rice leaves showing leaf spot symptoms collected throughout Peninsular Malaysia. Thirty-three isolates were recovered and identified based on their morphological characteristics such as conidia morphology, colony appearance, pigmentation and growth rate for species delimitation. Internal transcribed spacer (ITS) region was amplified to confirm the species identification. The 33 isolates were identified as Bipolaris sorokiniana (10 isolates), Curvularia hawaiiensis (8 isolates), C. geniculata (6 isolates), C. eragrostidis (6 isolates), C. aeria (2 isolates) and $C$. lunata ( 1 isolate). A phylogenetic tree was constructed based on ITS sequences using neighbour-joining method. The tree grouped members of Curvularia and Bipolaris into different clades. The phylogenetic tree indicated that the presence of two groups of fungi species; highly virulent and mild pathogens. In conclusion, Curvularia species and Bipolaris sorokiniana were present in rice field in Malaysia and associated with leaf spot of rice.
\end{abstract}

Keyword: Curvularia; Rice; Morphology; ITS region 